\title{
Standard Noether normalizations of the graph subring
}

\author{
Florian Luca $\cdot$ Rita Zuazua
}

Received: 22 June 2005 / Accepted: 27 March 2006 /

Published online: 11 July 2006

(C) Springer Science + Business Media, LLC 2006

\begin{abstract}
In this paper, we confirm a conjecture of Alcántar by showing that graph subrings always admit standard Noether normalizations.
\end{abstract}

Keywords Graph subring · Standard Noether normalizations

\section{Introduction}

Throughout this paper, $n>1$ is a positive integer, and $G$ is a simple graph with $n$ vertices which are labeled $1,2, \ldots, n$. We write $E(G):=\{\{i, j\} \mid i$ incident to $j\}$ for the set of edges of $G$, and $m$ for the cardinality of $E(G)$. We let $\mathbf{k}$ be a field of characteristic zero, or large enough with respect to $n$, and we write $R:=\mathbf{k}\left[x_{1}, \ldots, x_{n}\right]$ for the standard polynomial ring in $n$ indeterminates over $\mathbf{k}$. The monomial subring or the edge subring of $G$ (over $\mathbf{k}$ ) is the subring

$$
\mathbf{k}[G]:=\mathbf{k}\left[\left\{x_{i} x_{j} \mid\{i, j\} \in E(G)\right\}\right] \subset R .
$$

The presentation ideal $P(G)$ of $\mathbf{k}[G]$ is the kernel of the epimorphism of $\mathbf{k}$-algebras

$$
\psi: S=\mathbf{k}\left[\left\{t_{e} \mid e \in E(G)\right\}\right] \longrightarrow \mathbf{k}[G]
$$

induced by $\psi\left(t_{e}\right):=x_{i} x_{j}$ for $e=\{i, j\} \in E(G)$, where $\left\{t_{e}\right\}$ is a new set of variables in one to one correspondence with the edges of $G$. Notationally, we shall find it

Dedicated to the memory of Professor Victor Neumann Lara.

F. Luca $(\bowtie) \cdot$ R. Zuazua

Mathematical Institute, UNAM, Ap. Postal 61-3 (Xangari), CP 58 089, Morelia, Michoacán, Mexico

e-mail: fluca@matmor.unam.mx

R. Zuazua

e-mail: zuazua@matmor.unam.mx 
convenient to refer to the indeterminates $t_{e}$ for $e \in E(G)$ as $t_{i j}$, for $i \neq j$, with the convention that $t_{i j}$ is defined precisely when $i$ is incident to $j$, and in this case we do not distinguish between $t_{i j}$ and $t_{j i}$.

A Noether normalization of $\mathbf{k}[G]$, or, equivalently, of $S / P(G)$, is an integral extension of the form

$$
\mathbf{k}\left[h_{1}, \ldots, h_{d}\right] \longrightarrow S / P(G)
$$

where $h_{1}, \ldots, h_{d}$ are homogeneous polynomials in the $t_{i j}$ 's and $d$ is the Krull dimension of $\mathbf{k}[G]$. When all the $h_{\ell}$ 's for $\ell=1, \ldots, d$ are linear forms in the indeterminates $t_{i j}$, then the above Noether normalization is called standard.

The subring $\mathbf{k}[G]$, its presentation ideal $P(G)$, as well as the Noether normalizations for $\mathbf{k}[G]$ have been studied in [1, 4], and [5] and Chapter 6 of [7].

In [1], Alcántar asked if a standard Noether normalization of $\mathbf{k}[G]$ of the form

$$
h_{i}:=\sum_{j} a_{i j} t_{i j} \quad \text { with } a_{i j} \in \mathbf{k}, \quad \text { for } i=1, \ldots, n
$$

always exists provided that $\mathbf{k}[G]$ has Krull dimension $n$. Note that $k[G]$ has Krull dimension $n-1$ when $G$ is bipartite.

In this paper, we give an affirmative answer to the above question. We shall keep the exposition at an elementary level, and as such we avoid getting into technical issues such as Krull dimension, normality, or Cohen-Macaulay. We merely note that when $d=n$, the above question becomes equivalent to the following:

Question 1.1 ([1]). Is it true that there exist $2 m$ coefficients $a_{i j} \in \mathbf{k}$, for $(i, j) \in E(G)$, so that if we write

$$
h_{i}:=\sum_{\substack{1 \leq j \leq n \\\{i, j\} \in E(G)}} a_{i j} t_{i j}, \quad i=1, \ldots, n,
$$

then whenever $P$ is a prime ideal in $S$ containing $P(G)$ and $h_{i}$ for $i=1, \ldots, n$, then $P$ contains all the indeterminates $t_{i j}$ ?

We note that since $\mathbf{k}$ is of characteristic zero, it follows that $\mathbf{k}$ contains the field of rational numbers $\mathbf{Q}$, and, in particular, it also contains the ring of integers $\mathbf{Z}$.

Our result is:

Theorem 1.2. Let $n$ and $m \leq n(n-1) / 2$ be positive integers, and let $p$ be either zero or a sufficiently large prime with respect to $n$. Let $G$ be any graph with $n$ vertices and $m$ edges. Then there exist $2 m$ positive integers $a_{i j} \leq 2^{n+m}$ for $\{i, j\} \in E(G)$, such that if $\mathbf{k}$ is any field of characteristic zero or $p$, and $h_{i}$ are the linear forms in $S$ shown in (1.5), then the only prime ideal in $S$ containing $P(G)$ and $h_{i}$ for $i=1, \ldots, n$ is the maximal ideal containing all the indeterminates $t_{i j}$.

As one of the referees observed, the above Theorem 1.2 can be formulated in terms of special semigroup rings over a field. Namely, let $S$ be a semigroup ring of the Springer 
polynomial ring $R$ generated by squarefree monomials of degree 2 , let $E_{i}$ be the generators of $S$ which are divisible by $x_{i}$, and let $h_{i}$ be a general linear combination of the elements $E_{i}$ for $i=1, \ldots, n$. Then Theorem 1.2 says (a little bit more than) that the radical of $\left(h_{1}, \ldots, h_{n}\right)$ is the maximal homogeneous ideal of $S$. Formulated in this way, the statement makes sense for any general (affine) semigroup rings. We have neither investigated this problem in the above generality, nor do we know of any counter-examples to the general statement, but would like to pose this as an open problem.

Throughout the rest of this paper, we assume that $G$ is a graph without isolated vertices (i.e., degree zero vertices). Except for the last section, we also assume that the characteristic of $\mathbf{k}$ is zero, and we prove Theorem 1.2 in this instance. In the last section, we comment about the case when $\mathbf{k}$ has a large characteristic and sketch the proof of Theorem 1.2 in this instance. We note that the restriction that $G$ has no isolated vertices imposes no restriction on our problem for if $i_{0} \in V(G)$ is an isolated vertex of $G$, then none of the degree two monomials $x_{i} x_{j}$ for $\{i, j\} \in E(G)$ generating $\mathbf{k}[G]$ as a subring of $R$ involves the indeterminate $x_{i_{0}}$, and therefore $\mathbf{k}[G]$ lives in $\mathbf{k}\left[x_{i} \mid i \neq i_{0}\right]$. We shall also assume that the coefficients $a_{i j}$ for $\{i, j\} \in E(G)$ appearing in (1.5) are nonzero.

The rest of the paper is organized as follows. In Section 2, we associate to $G$ an $n \times n$ matrix $A_{G}$ with entries in $\mathbf{k}$ and whose entry $(i, j)$ is nonzero if and only if $\{i, j\} \in E(G)$, and ask the nullspace of such a matrix to satisfy certain special properties. In Section 3, we give necessary and sufficient conditions on the set of linear forms $\left\{h_{1}, \ldots, h_{n}\right\}$ defined in (1.5) to be a standard Noether normalization in terms of the matrices with special properties described in Section 2. In Section 4, we treat the matrices from Section 2 as "generic", i.e., with algebraically independent nonzero entries, and we show that such matrices do fulfill the special properties described in Section 2. In Section 5, we use the results from Sections 2 and 4, to prove that for each graph $G$ there exists a universal polynomial in $2 m$ indeterminates of degree at most $2^{n+m}$ and with integer coefficients, such that if the vector of coefficients $\mathbf{a}:=$ $\left(a_{i j} \mid\{i, j\} \in E(G)\right) \in \mathbf{Z}^{2 m}$ is not a zero of such a polynomial, then the matrix defined in Section 2 and whose nonzero $(i, j)$ entry is precisely the component $a_{i j}$ of the above vector a has the required special properties described in Section 2. In Section 6, we give the proof of Theorem 1.2, which will by then be just a straightforward consequence of the results from the previous sections. Section 7 contains some comments and remarks as well as the proof of Theorem 1.2 when $p$ is sufficiently large with respect to $n$.

\section{Some matrices associated to graphs}

Given a graph $G$ with $n$ vertices labeled $1, \ldots, n, m$ edges, and without isolated points, we write $A_{G}$ for an $n \times n$ matrix with entries in $\mathbf{k}$ such that $a_{i j} \neq 0$ if and only if $\{i, j\} \in E(G)$. Note that $A_{G}$ is not necessarily symmetric, although if $i \neq j$, then $a_{i j}=0$ if and only if $a_{j i}=0$. Conversely, given a matrix $A \in M_{n}(\mathbf{k})$, this matrix equals $A_{G}$ for some graph $G$ as above if and only if the set of its zero entries is a relation $\mathcal{R}_{A}$ on $\{1, \ldots, n\}^{2}$, which is reflexive, symmetric, has the property that for every $i \in\{1, \ldots, n\}$ there exists $j \in\{1, \ldots, n\}$ such that $(i, j) \notin \mathcal{R}_{A}$, and $\mathcal{R}_{A}$ consists of $n^{2}-2 m$ elements. Note that changing the labeling of the vertices permutes the rows 
of the matrix $A_{G}$. In what follows, whenever we refer to $A_{G}$ we mean that some labeling of the vertices of $G$ has been fixed.

Definition 2.1. A matrix $A \in M_{n}(\mathbf{k})$ is called a zero component kernel matrix (or $Z C K$, for short), if for all $\mathbf{v}:=\left(v_{1}, \ldots, v_{n}\right) \in \operatorname{ker}(A)$ there exists $i \in\{1, \ldots, n\}$ such that $v_{i}=0$.

Note that if $A$ is a $Z C K$ matrix in $M_{n}(\mathbf{k})$, then there exists $i \in\{1, \ldots, n\}$ such that the containment $\operatorname{ker}(A) \subseteq V_{i}:=\left\{v \in \mathbf{k}^{n} \mid v_{i}=0\right\}$ holds, provided that either $\mathbf{k}$ is of characteristic zero (hence, infinite), or $\mathbf{k}$ contains more than $n$ elements.

Let $G$ be a graph and let $H$ be a subgraph of $G$ without isolated points. If $A:=A_{G}$ is a matrix associated to $G$ as above, we write $A \downarrow_{H}^{G}$ for the restriction of $A$ to $H$, i.e., for the matrix associated to $H$ and induced by $A$. Since $H$ is obtained from $G$ by first removing a subset $E^{\prime} \subset E(G)$ of edges of $G$, and then removing the subset of vertices $V^{\prime} \subset V(G)$ which have become isolated after removing $E^{\prime}$, it follows that $A \downarrow_{H}^{G}$ is obtained from $A$ by first replacing the $(i, j)$ entry of $A$ by 0 for all $\{i, j\} \in E^{\prime}$, and then removing the $i$ th row and column for all $i \in V^{\prime}$ since these rows and columns have now become identically zero. Note also that given $E^{\prime}$, the subset $V^{\prime}$ of $V(G)$ is uniquely determined, and consists precisely of those vertices $i \in V(G)$ such that all edges adjacent to them are in $E^{\prime}$.

Definition 2.2. Let $G$ be a graph without isolated vertices and let $A:=A_{G} \in M_{n}(\mathbf{k})$ be a matrix associated to $G$ as above. We say that $A$ is a hereditarily zero component kernel matrix (or $H Z C K$, for short) if $A \downarrow_{H}^{G}$ is a $Z C K$ matrix for all subgraphs $H \subseteq G$ of $G$ without isolated vertices.

We recall that a subset $V^{\prime} \subset V(G)$ of vertices of $G$ is called a vertex cover for $G$ if for all $j \in V(G)$ there exists $i \in V^{\prime}$ such that $(i, j) \in E(G)$.

Definition 2.3. Let $G$ be a graph without isolated vertices and let $A:=A_{G} \in M_{n}(\mathbf{k})$ be a matrix associated to $G$ as above. We say that $A$ is a vertex cover zero component kernel matrix (or $V C Z C K$, for short), if whenever $\mathbf{v} \in \operatorname{ker}(A)$, the set $V^{\prime}:=\{i \in$ $\left.V(G) \mid v_{i}=0\right\}$ is a vertex cover for $G$.

Given a graph $G$ with $n$ vertices none of which is isolated, and $A:=A_{G}$ a matrix associated to $G$ as above, it is clear that if $A$ is $H Z C K$, then it is $Z C K$ as well. The main result of this section is the following:

Proposition 2.4. Let $A \in M_{n}(\mathbf{k})$ be a matrix associated to a graph $G$ without isolated vertices. If $A$ is $H Z C K$, then it is VCZCK as well.

Proof of Proposition 2.4. Let $\mathbf{v} \in \operatorname{ker}(A)$. Since $A$ is $H Z C K$ (in particular, $Z C K$ ), a vertex $i \in V(G)$ exists such that $v_{i}=0$. Let $G^{\prime}$ be the subgraph of $G$ obtained by first eliminating the vertex $i$ and all edges adjacent to it, and then also removing all vertices which have now become isolated. If $G^{\prime}$ is empty, then $\{i\}$ is already a vertex cover for $G$, and we are done. If not, write $V^{\prime}:=V\left(G^{\prime}\right), n^{\prime}:=\left|V^{\prime}\right|$ and $A^{\prime}:=A \downarrow_{G^{\prime}}^{G} \in M_{n^{\prime}}(\mathbf{k})$. Note that $V \backslash V^{\prime}$ consists of $i$ and eventually some other vertices all of which are Springer 
adjacent only to $i$. Let $\mathbf{v}^{\prime}:=\mathbf{v} \downarrow_{G^{\prime}}^{G}$ be the vector in $\mathbf{k}^{n^{\prime}}$ which is the projection of $\mathbf{v}$ on the subspace of those coordinates whose indexes are in $V^{\prime}$. The equation $A \cdot \mathbf{v}^{T}=0$ together with the fact that $v_{i}=0$ imply that $A^{\prime} \cdot \mathbf{v}^{\prime T}=0$, and therefore $\mathbf{v}^{\prime} \in \operatorname{ker}\left(A^{\prime}\right)$. Since $A$ is $H Z C K$, it follows that there exists $i^{\prime} \in V^{\prime}$ such that $v_{i^{\prime}}^{\prime}=0$. In particular, $v_{i^{\prime}}=0$. Proceeding in this way, after a finite number of steps we arrive at the conclusion that there exists a subset $I$ of $V(G)$ such that $v_{i}=0$ for all $i \in I$, and such that $I$ is a vertex cover for $G$, which completes the proof of Proposition 2.4.

\section{Matrices associated to graphs and standard Noether normalizations}

In this section, we give necessary and sufficient conditions for the set of forms $\left\{h_{1}, \ldots, h_{n}\right\}$ shown in (1.5) to be a standard Noether normalization, in the sense of fulfilling the conditions from Question 1.1, in terms of the matrix $A:=A_{G}\left(h_{1}, \ldots, h_{n}\right)$ whose rows are the normal vectors to the forms $h_{i}$ for $i=1, \ldots, n$.

Proposition 3.1. Let $h_{i}$ be the forms defined in (1.5) for $i=1, \ldots, n$. Assume that $a_{i j} \neq 0$ for all $\{i, j\} \in E(G)$. Let $A$ be the matrix whose ith row is the normal vector to the form $h_{i}$ for all $i=1, \ldots, n$. If $A$ is not $V C Z C K$, then there exists a prime ideal $P$ in $S$ containing $P(G)$ and $h_{i}$ for all $i=1, \ldots, n$ but which does not contain $t_{e}$ for all $e \in E(G)$.

Proof of Proposition 3.1. We look at the system of linear equations $A \cdot \mathbf{v}^{T}=0$, where the unknowns are the components of $\mathbf{v}:=\left(v_{1}, \ldots, v_{n}\right)$. Since $A$ is not $V C Z C K$, it follows, in particular, that $A$ is degenerate. Thus, there exists a partition $I \cup J$ of $\{1, \ldots, n\}$ into two nonempty disjoint subsets and $L_{i}: \mathbf{k}^{|J|} \longmapsto \mathbf{k}$ for $i \in I$, that are either linear forms, or constant zero maps, such that all solutions of the above system have $v_{i}=L_{i}\left(\mathbf{v}_{J}\right)$, where we write $\mathbf{v}_{J}:=\left(v_{j} \mid j \in J\right)$. Let $I_{1} \subset I$ be the set of indices $i$ in $I$ such that $L_{i} \equiv 0$. The condition that $A$ is not $V C Z C K$ together with the fact that $\mathbf{k}$ is infinite (which guarantees that for every finite number of linear forms on some finite dimensional vector space over $\mathbf{k}$ there exists a vector which lies outside the union of the kernels of these forms) implies that there exist indices $\ell$ and $s$ in $V(G) \backslash I_{1}$ such that $(\ell, s) \in E(G)$, but neither $\ell$ nor $s$ is adjacent with any vertex in $I_{1}$. Let $\mathcal{I}$ be the ideal of $R$ generated by the forms $x_{i}-L_{i}\left(\mathbf{x}_{J}\right)$ for $i \in I$, where $\mathbf{x}_{J}:=\left(x_{j} \mid j \in J\right)$. We claim that there exists a minimal prime ideal $Q$ of $R$ containing $\mathcal{I}$ and such that $x_{\ell} x_{s}$ does not belong to $Q$. Indeed, assume that this is not the case. It then follows that there exists a positive integer $N$ such that the relation

$$
\left(x_{\ell} x_{s}\right)^{N}=\sum_{i \in I} f_{i}\left(x_{i}-L_{i}\left(\mathbf{x}_{J}\right)\right)
$$

holds in $R$ with some polynomials $f_{i}:=f_{i}\left(x_{1}, \ldots, x_{n}\right) \in R$. Consider the totality of $\left|I \backslash I_{1}\right|+|J| \leq n$ linear forms on $\mathbf{k}^{|J|}$ given by $L_{i}^{\prime}(\mathbf{w}):=L_{i}(\mathbf{w})$ for $i \in I \backslash I_{1}$, and $L_{j}^{\prime}(\mathbf{w}):=w_{j}$ for $j \in J$, where $\mathbf{w}:=\left(w_{j} \mid j \in J\right)$. Since $\mathbf{k}$ is infinite, a vector $\mathbf{w}^{(0)} \in$ $\mathbf{k}^{|J|}$ exists such that none of the above linear forms vanishes at $\mathbf{w}^{(0)}$. We then specialize relation (3.1) with $x_{i}:=0$ for $i \in I_{1}, x_{j}:=w_{j}^{(0)}$ for $j \in J$, and $x_{i}:=L_{i}\left(\mathbf{w}^{(0)}\right)$ for $i \in$ 
$I \backslash I_{1}$, to obtain that $\left(x_{\ell} x_{s}\right)^{N}=0$ for this particular specialization, which is impossible from the way we have constructed the point $\mathbf{w}^{(0)} \in \mathbf{k}^{|J|}$.

Thus, there does indeed exist an ideal $Q$ of $R$ containing $\mathcal{I}$ and not containing $x_{\ell} x_{s}$. From the construction, it is clear that

$$
\sum_{\substack{1 \leq j \leq n \\\{i, j\} \in E(G)}} a_{i j} x_{j} \in \mathcal{I} \subset \mathcal{Q}
$$

holds for all $i \in\{1, \ldots, n\}$. Consider the ideal $Q^{\prime}:=Q \cap \mathbf{k}[G]$ of $\mathbf{k}[G]$. This is a prime ideal in $\mathbf{k}[G]$, which does not contain the monomial $x_{\ell} x_{s}$, but does contain the linear forms in $\mathbf{k}[G]$ given by

$$
\sum_{\substack{1 \leq j \leq n \\\{i, j\} \in E(G)}} a_{i j} x_{i} x_{j}
$$

for all $i \in\{1, \ldots, n\}$. Writing $P:=\psi^{-1}\left(Q^{\prime}\right)$, we have obtained a prime ideal $P$ of $S$ containing $P(G)$ and $h_{i}$ for all $i=1, \ldots, n$, and not containing $t_{\ell s}$, which completes the proof of Proposition 3.1.

Remark 3.2. We note that the above arguments remain valid when $\mathbf{k}$ is any field with more than $n$ elements.

Proposition 3.3. Let $h_{i}$ be the forms shown in (1.5), and let $A \in M_{n}(\mathbf{k})$ be the matrix whose $i$ th row is the normal vector to $h_{i}$ for all $i=1, \ldots, n$. Assume that $A$ is $H Z C K$. Then every prime ideal $P$ of $S$ containing $P(G)$ and $h_{i}$ for $i=1, \ldots, n$, contains $t_{e}$ for all $e \in E(G)$.

Proof of Proposition 3.3: We use induction on the size $n+m$ of the graph $G$. Suppose first that $G$ is a tree. Then there exist a vertex of degree 1 in $V(G)$. Up to relabeling the vertices, we may assume that this vertex is $n$. Then there exists a unique vertex $i \in\{1, \ldots, n-1\}$ such that $n$ is adjacent to $i$. Since $h_{n}=a_{n i} t_{n i}$, we get that if $P$ is a prime ideal of $S$ containing $P(G)$ and $h_{i}$ for $i=1, \ldots n$, then since $a_{n i} \neq 0$, it follows that $t_{n i} \in P$. Moreover,

$$
\sum_{\substack{1 \leq j \leq n-1 \\\{i, j\} \in E(G)}} a_{i j} t_{i j}
$$

belongs to $P$ as well. Thus, we have reduced the problem to the subgraph $G \backslash\{e\}$ of $G$, where $e:=\{i, n\}$, which is also a tree with $n-1$ vertices. Thus, the proof of Proposition 3.3 for the case of trees follows by an easy induction on $n$, the case $n=2$ proved by the above argument. Note that only the fact that $a_{i j} \neq 0$ for all $\{i, j\} \in E(G)$ is needed in the case when $G$ is a tree (and that $P(G)=0$ in this case). In particular, the assertion of Proposition 3.3 follows for all graphs $G$ without isolated vertices and with $n+m \leq 5$.

Assume now that $G$ is some graph without isolated vertices and that the assertion of Proposition 3.3 holds for all subgraphs of sizes smaller than the size of $G$. Assume, Springer 
to the contrary, that the forms $h_{i}$ for $i=1, \ldots, n$ are such that the matrix associated to these forms is $H Z C K$, but that a prime ideal $P$ of $S$ exists which contains $P(G)$ and all the forms $h_{i}$ for $i=1, \ldots, n$, but which does not contain some of $t_{e}$ for $e \in E(G)$. At this point, we claim that we can assume even more about $P$, namely that it does not contain any of the edge indeterminates $t_{e}$ for $e \in E(G)$. Indeed, assume that $P$ contains $t_{e}$ for some $e:=\{\ell, s\} \in E(G)$. Let $G_{1}$ be the graph obtained from $G \backslash\{e\}$ by removing the isolated vertices (which can only be $\ell$, or $s$, or both). Let $V_{1}$ be the set of vertices of $G_{1}$. Since $P$ contains $t_{e}$ and $h_{i}$ for all $i=1, \ldots, n$, it follows that $P$ contains as well the linear forms $h_{i} \downarrow_{G_{1}}^{G}$ for all $i=1, \ldots, n$, where we define the above form to equal $h_{i}$ if $i \notin\{\ell, s\}$, and to equal $h_{i}-a_{i j} t_{i j}$ if $i \in\{\ell, s\}$, where in this last case we use $j$ for the only vertex in $V(G)$ such that $\{i, j\}=\{\ell, s\}$. Note that $h_{i} \downarrow_{G_{1}}^{G}$ is zero if and only if $i \notin V_{1}$. The matrix associated to the nonzero forms $h_{i} \downarrow_{G_{1}}^{G}$ is precisely $A \downarrow_{G_{1}}^{G}$. Since $A$ is $H Z C K$, it follows that $A \downarrow_{G_{1}}^{G}$ is also $H Z C K$, and now the induction hyphotesis applied to $G_{1}$ shows that $P$ must indeed contain $t_{f}$ for all $f \in E\left(G_{1}\right)$. Since $E(G)=E\left(G_{1}\right) \cup\{e\}$, we do get that $P$ contains $t_{f}$ for all $f \in E(G)$.

Thus, we shall assume that $P$ does not contain any $t_{e}$ for $e \in E(G)$. We now note that this assumption together with the induction hypothesis shows that $G$ is connected and that every vertex has degree $\geq 2$. Indeed, if $G$ is not connected, then, up to relabeling the vertices of $G$, the matrix $A$ is seen as being a diagonal block matrix whose diagonal blocks are $A \downarrow_{G_{i}}^{G}$, where $G_{1}, \ldots, G_{t}$ are the connected components of $G$ and $t \geq 2$. Clearly, $A \downarrow_{G_{i}}^{G}$ is the matrix whose rows are just the normal vectors to $h_{\ell}$ for $\ell \in V\left(G_{i}\right)$ and for $i=1, \ldots, t$. By the induction hypothesis applied to each one of the connected components $G_{i}$ of $G$ for $i=1, \ldots, t$, we get that $P$ must indeed contain all $t_{e}$ for $e \in E(G)$. The fact that $G$ does not contain any vertex of degree 1 is follows by the argument for the case of trees together with the fact that $a_{i j} \neq 0$ for $\{i, j\} \in$ $E(G)$.

In the above two instances, when we invoked the induction hypothesis, we had in mind the following argument. Assume that $H$ is a nonempty proper subgraph of $G$ without isolated points such that either $t_{f} \in P$ for all $f \in V(G) \backslash V(H)$, or $H$ is the union of some (but not all) connected components of $G$, if $G$ is not connected. Since $h_{i} \in P$ holds for all $i=1, \ldots, n$, it follows easily that under the above assumptions $h_{i} \downarrow_{H}^{G} \in P$ holds for all $i=1, \ldots, n$. The matrix $A \downarrow_{H}^{G}$ is $H Z C K$ and its rows are precisely the normal vectors to the nonzero forms $h_{i} \downarrow_{H}^{G}$, and by the induction hypothesis every prime ideal in $S^{\prime}:=\mathbf{k}\left[t_{e} \mid e \in V(H)\right]$ containing $h_{i} \downarrow_{H}^{G}$ and $P(H)$ contains $t_{e}$ for all $e \in V(H)$ as well. This shows that the radical of the ideal $\mathcal{J}_{H}$ generated by $h_{i} \downarrow_{H}^{G}$ for $i=1, \ldots, n$ and $P(H)$ in $S^{\prime}$ is precisely $\left(t_{e} \mid e \in V(H)\right) \cdot S^{\prime}$. Thus, there exists a positive integer $N$ such that for all $e \in V(H) t_{e}^{N}$ is a linear combination with coefficients in $S^{\prime}$ of $h_{i} \downarrow_{H}^{G}$ for $i=1, \ldots, n$ and elements of $P(H)$ for all $e \in V(H)$. Since $S^{\prime} \subset S, P(H) \subset P(G) \subset P$, and $h_{i} \downarrow_{H}^{G} \in P$ for $i=1, \ldots, n$, we obtain that $t_{e}^{N} \in P$ for all $e \in V(H)$, and therefore $t_{e} \in P$ holds for all $e \in V(H)$ as well.

We now leave this prime ideal $P$ for the time being, we let $\mathcal{J}$ be the ideal of $\mathbf{k}[G]$ generated by

$$
\sum_{\substack{1 \leq j \leq n \\\{i, j\} \in E(G)}} a_{i j} x_{i} x_{j}
$$


for $i=1, \ldots, n$, and we write $\mathcal{I}$ for the extension of $\mathcal{J}$ to $R$. Note that $\mathcal{I}$ is generated by the same elements as the ones shown in (3.2). As such, it is an ideal contained in the Rees ideal $\mathcal{I}(G):=\left(x_{i} x_{j} \mid\{i, j\} \in E(G)\right) \cdot R$ of $R$. We now claim that if $Q$ is a minimal prime ideal containing $\mathcal{I}$, then $Q$ contains $\mathcal{I}(G)$ as well. To see this, let $Q$ be such a prime ideal. Writing $\overline{x_{i}}$ for the class of $x_{i}$ modulo $Q$, we get the system of quadratic equations

$$
\overline{x_{i}} \sum_{\substack{1 \leq j \leq n \\\{i, j\} \in E(G)}} a_{i j} \overline{x_{j}} .
$$

in the domain $R / Q$. The above system of Eqs. (3.3) together with the fact that $R / Q$ is a domain implies that there exist disjoint subsets $I_{1}$ and $I_{2}$ of $V(G)$ (one of them might be empty) whose union is $V(G)$, and such that

$$
\overline{x_{i}}=0 \quad \text { for all } i \in I_{1} \text {, }
$$

and

$$
\sum_{\substack{1 \leq j \leq n \\\{i, j\} \in E(G)}} a_{i j} \overline{x_{j}}=0 .
$$

Let $G_{1}$ be the subgraph of $G$ obtained by removing from $G$ all the vertices $i \in I_{1}$ and all the edges adjacent to them, and then deleting the vertices which have become isolated. If $G_{1}$ is empty, it follows that $I_{1}$ is a vertex cover for $G$. Assume now that $G_{1}$ is nonempty, and write $V_{1}:=V\left(G_{1}\right)$. Note that $V_{1} \subset I_{2}$. Injecting relations (3.4) into (3.5), and eliminating the trivial equations produced in this way (corresponding to vertices in $\left.I_{2} \backslash V_{1}\right)$, we get that $\overline{\mathbf{x}}_{V_{1}}:=\left(\overline{x_{i}} \mid i \in V_{1}\right)$ is in the kernel of $A \downarrow_{G_{1}}^{G}$ regarded as an element of $M_{\left|V_{1}\right|}(R / Q)$. However, $A \downarrow_{G_{1}}^{G}$ is an $H Z C K$ matrix as an element of $M_{\left|V_{1}\right|}\left(\mathbf{k}^{\left|V_{1}\right|}\right)$, and from the structure of the kernel of any square matrix, it follows easily that $A \downarrow_{G_{1}}^{G}$ is also a $H Z C K$ matrix over any integral domain which is a k-algebra (because $\operatorname{ker}_{P / Q}\left(A \downarrow_{G_{1}}^{G}\right)$ is simply obtained from $\operatorname{ker}_{\mathbf{k}}\left(A \downarrow_{G_{1}}^{G}\right)$ by extending the scalars to $R / Q$ ). Thus, by Proposition 2.4, we arrive at the conclusion that there exists a vertex cover $I_{3}$ of $G_{1}$ such that $\overline{x_{i}}=0$ for all $i \in I_{3}$. Since $I_{1} \cup I_{3}$ is a vertex cover for $G$, we conclude that we have just proved that the system of Eq. (3.3) in $R / Q$ implies that there exists a vertex cover $I$ of $G$ such that $\overline{x_{i}}=0$ for all $i \in I$. Interpreting this in $R$, we conclude that $x_{i} x_{j} \in Q$ holds for all $\{i, j\} \in E(G)$, and therefore $\mathcal{I}(G) \subset Q$. Since this is true for all prime ideals $Q$ of $R$ containing $\mathcal{I}$, we get that $\operatorname{rad}(\mathcal{I})=\operatorname{rad}(\mathcal{I}(G))$, and since $R$ is Noetherian we conclude that $\mathcal{I}(G)^{N} \subset \mathcal{I}$.

We now pick an arbitrary edge $\{\ell, s\}$ of $G$ and conclude that there must exist a relation of the form

$$
\left(x_{\ell} x_{s}\right)^{N}=\sum_{1 \leq i \leq n} f_{i} \psi\left(h_{i}\right)
$$

with $f_{i}:=f_{i}\left(x_{1}, \ldots, x_{n}\right) \in R$. We may assume that $N \geq 2$. Note that if it were true that all the polynomials $f_{i}$ appearing in (3.6) can be represented as polynomials in Springer 
the monomials $x_{i} x_{j}$ for $\{i, j\} \in E(G)$, then we would immediately get that $\left(x_{\ell} x_{s}\right)^{N} \in$ $\psi(P)$, which contradicts the fact that $P$ does not contain any of the $t_{e}$ for $e \in E(G)$. We also notice that since $\left(x_{\ell} x_{s}\right)^{N}$ is homogeneous of degree $2 N$ and $\psi\left(h_{i}\right)$ is homogeneous of degree 2 for $i=1, \ldots, n$, it follows that we may assume that all the polynomials $f_{i}$ appearing in (3.6) are homogeneous of degree $2 N-2$ for all $i=1, \ldots N$.

From now on, we distinguish two cases according to the geometry of $G$. Note that since $G$ is connected and since every vertex of it is of degree $\geq 2$, it follows that $G$ cannot be a tree, and as such it contains cycles.

Case 1. $G$ contains an odd cycle.

Recall that a path in $G$ is just a concatenation of edges $e_{1} \ldots e_{t}$ where $e_{\lambda}:=\left\{i_{\lambda}, i_{\lambda+1}\right\} \in$ $E(G)$ for all $\lambda=1, \ldots, t$. We call $i_{1}$ the starting point and $i_{t+1}$ the final point of the path, respectively. We say that $t$ is the length of the path, and that the path is a circuit if $i_{1}=i_{t+1}$.

Since $G$ contains an odd cycle, it follows that for all $i, j$ in $V(G)$ (not necessarily distinct) there exists an odd path from $i$ to $j$. If this path is $e_{1} \ldots e_{t}$ with $t$ odd, it is then immediate that

$$
x_{i} x_{j}=\prod_{\lambda=1}^{t} M_{e_{\lambda}}^{(-1)^{\lambda-1}}
$$

where we write $M_{e}:=x_{i_{e}} x_{j_{e}}$ for every $e:=\left\{i_{e}, j_{e}\right\} \in E(G)$. Formula (3.7) shows therefore that every degree two monomial $x_{i} x_{j}$ is a ratio of products of edge monomials; i.e., those degree two monomials which appear in the list of generators of $\mathcal{I}(G)$. In particular, every monomial of even degree in $R$ is also a ratio of products of edge monomials, and since each one of the polynomials $f_{i}$ appearing in (3.6) is homogeneous of even degree $2 N-2$, we conclude that each one of the polynomials $f_{i}$ is a linear combination of fractional monomials in the edge monomials. This shows that there exists a positive integer $M$ such that

$$
\left(\prod_{\{i, j\} \in E(G)} x_{i} x_{j}\right)^{M} \cdot\left(x_{\ell} x_{s}\right)^{N}=\sum_{i=1}^{n}\left(\prod_{\{i, j\} \in E(G)} x_{i} x_{j}\right)^{M} \cdot f_{i} \cdot \psi\left(h_{i}\right),
$$

and such that for each $i=1, \ldots, n$ the expression

$$
F_{i}=\left(\prod_{\{i, j\} \in E(G)} x_{i} x_{j}\right)^{M} \cdot f_{i}
$$

is a linear combination of monomials in the edge monomials $x_{i} x_{j}$ for $\{i, j\} \in E(G)$. In particular, the relation

$$
\left(\prod_{\{i, j\} \in E(G)} x_{i} x_{j}\right)^{M} \cdot\left(x_{\ell} x_{s}\right)^{N}=\sum_{i=1}^{n} F_{i} \cdot \psi\left(h_{i}\right)
$$


is a relation in $\mathbf{k}[G]$ and shows that

$$
\left(\prod_{\{i, j\} \in E(G)} x_{i} x_{j}\right)^{M} \cdot\left(x_{\ell} x_{s}\right)^{N} \in \mathcal{J}
$$

Thus, the prime ideal $\psi(P)$ of $\mathbf{k}[G]$ contains the element appearing on the left hand side of relation (3.9), and since $\psi(P)$ is prime, it follows that there exist $i$ and $j$ with $\{i, j\}=e \in E(G)$ such that $x_{i} x_{j} \in \psi(P)$. This implies that $t_{e} \in P$, which is the desired contradiction.

Case 2. $G$ does not contain an odd cycle.

In this case $G$ is bipartite. Let $I \cup J=V(G)$ be a partition of the set of vertices of $G$ such that all edges of $G$ are of the form $\{i, j\}$ for some $i \in I$ and $j \in J$. We shall call a monomial $M:=\prod_{i=1}^{n} x_{i}^{\alpha_{i}} \in R$ balanced if $\sum_{i \in I} \alpha_{i}=\sum_{j \in J} \alpha_{j}$; i.e., if its total $I$-degree equals its total $J$-degree. Note that the product of two balanced monomials is a balanced monomial, while the product of a balanced monomial with an unbalanced one is unbalanced. Relation (3.6) together with the fact that $\left(x_{\ell} x_{s}\right)^{N}$ is balanced when $\{\ell, s\} \in E(G)$, as well as with the fact that $\psi\left(h_{i}\right)$ is balanced for all $i=1, \ldots, N$, show that we may assume that all monomials which effectively appear in $f_{i}$ are balanced of degree $2 N-2$. In particular, every monomial which appears in anyone of the $f_{i}$ 's is of the form $\prod_{\lambda=1}^{N-1}\left(x_{i_{\lambda}} x_{j_{\lambda}}\right)$ where $i_{\lambda} \in I$ and $j_{\lambda} \in J$ for all $\lambda=1, \ldots, N-1$.

We now note that for all $i \in I$ and $j$ in $J$ there exists a path in $G$ from $i$ to $j$ which is necessarily odd. This shows that formula (3.7) holds in this case as well whenever $i \in I$ and $j \in J$. The same argument as the one employed in the previous case now shows that containment (3.9) holds, and, in particular, that $\psi(P)$ must indeed contain $x_{i} x_{j}$ for some $\{i, j\}=e \in E(G)$. Thus, $t_{e}$ must belong to $P$, which is again a contradiction, and completes the proof of Case 2 and of Proposition 3.3.

Remark 3.4. A different proof for the case when $G$ contains an odd cycle can be achieved as follows. Assume that $P$ is a prime ideal of $S$ containing $P(G)$ and $h_{i}$ for $i=1, \ldots, n$, but $t_{e} \notin P$ for all $e \in E(G)$. Then $\psi(P)$ is a prime ideal of $\mathbf{k}[G]$ which is disjoint from the multiplicative system $\mathcal{U}$ of $\mathbf{k}[G]$ generated by $x_{i} x_{j}$ for $\{i, j\} \in E(G)$. Invert the elements of $\mathcal{U}$ to obtain that $\psi(P) \cdot \mathcal{U}^{-1}$ is a prime ideal of $\mathbf{k}[G] \cdot \mathcal{U}^{-1} \subset$ $R \cdot \mathcal{U}^{-1}$. Relation (3.7) for $j=i$ shows that $x_{i} / 1 \in R \cdot \mathcal{U}^{-1}$ is a quadratic integer over $\mathbf{k}[G] \cdot \mathcal{U}^{-1}$. Since this is true for all $i=1, \ldots, n$, we conclude that the extension $\mathbf{k}[G] \cdot \mathcal{U}^{-1} \subset R \cdot \mathcal{U}^{-1}$ is integral. The Going Up Theorem can therefore be applied and leads to the conclusion that there exists a prime ideal $Q^{\prime}$ of $R \cdot \mathcal{U}^{-1}$ sitting above $\psi(P) \cdot \mathcal{U}^{-1}$. This prime ideal is certainly of the form $Q \cdot \mathcal{U}^{-1}$ for some prime ideal $Q$ of $R$ containing $\psi(P)$ and disjoint from $\mathcal{U}$. Since $Q$ contains $\psi(P)$, it follows that $Q \supset \mathcal{I}$. But we have just seen that this implies that $Q$ contains $\mathcal{I}(G)$, and in particular it contains all elements from $\mathcal{U} \backslash\{1\}$, which is a contradiction.

Unfortunately, we could not find an analogue of this shorter argument for the case in which $G$ does not contain an odd cycle. 


\section{Nullspaces of zero symmetric and zero diagonal square matrices with algebraically independent nonzero entries}

This section is independent of the preceding ones.

Throughout this section, we let $\mathbf{k}$ to be any field, $n \geq 2$ to be any positive integer, and $\mathbf{K} \supset \mathbf{k}$ to be a field extension of $\mathbf{k}$ of a sufficiently large transcendence degree (say, at least $n^{2}$ ).

Let $A_{n}(\mathbf{K})$ be the subset of $M_{n}(\mathbf{K})$ consisting of all $n \times n$ matrices $A=\left(a_{i j}\right)_{1 \leq i, j \leq n}$ with entries in $\mathbf{K}$ which fulfill the following properties:

1. $a_{i i}=0$ for all $i=1, \ldots, n$.

2. $a_{i j}=0$ if and only if $a_{j i}=0$.

3. For all $i=1, \ldots, n$ there exists $j$ so that $a_{i j} \neq 0$.

4. The nonzero entries $a_{i j}$ are algebraically independent over $\mathbf{k}$.

Put $V:=\mathbf{K}^{n}$ and for $i=1, \ldots, n$ put $V_{i}:=\left\{\mathbf{v}=\left(v_{1}, \ldots, v_{n}\right) \in V \mid v_{i}=0\right\}$. Our main result in this section addresses the structure of $\operatorname{Ker}(A)$, when $A \in A_{n}(\mathbf{K})$.

Proposition 4.1. If $n \geq 2$ and $A \in A_{n}(\mathbf{K})$, then $\operatorname{Ker}(A) \subset V_{i}$ for some $i=1, \ldots, n$.

To make the above statement more precise, let $\mathcal{N}$ be a binary relation on $\{1,2, \ldots, n\}$ which is symmetric, reflexive, and has the property that for all $i$ there exists $j$ such that $(i, j) \notin \mathcal{N}$. Write $A_{n, \mathcal{N}}(\mathbf{K})$ for the set of all matrices $A$ in $M_{n}(\mathbf{K})$ such that $a_{i j}=0$ if $(i, j) \in \mathcal{N}$, and such that the remaining $n^{2}-|\mathcal{N}|$ entries of $A$ are algebraically independent over $\mathbf{k}$ (in particular, nonzero as well). Then,

$$
A_{n}(\mathbf{K})=\bigcup_{\mathcal{N}} A_{n, \mathcal{N}}(\mathbf{K})
$$

where in the above equation $\mathcal{N}$ runs over all the binary relations on $\{1,2, \ldots, n\}$ with the properties specified above. Proposition 4.1 then says that for all such $\mathcal{N}$ there exists $i$ such that if $A \in A_{n, \mathcal{N}}(\mathbf{K})$ and $\mathbf{v}$ is a nullvector of $A$, then its $i$ th component is zero.

First Proof of Proposition 4.1: Let $A \in A_{n, \mathcal{N}}$, and write $G$ for the graph on the set of vertices $[n]:=\{1,2, \ldots, n\}$ and whose edge set is the complement of $\mathcal{N}$ in $[n]^{2}$. We write $A_{G}$ for $A$. Note that, formally, $A_{G}$ is precisely the matrix whose rows are the normal vectors to the forms $h_{i}$ for $i=1, \ldots, n$ shown in (1.5), with the only difference that the entries $a_{i j}$ for $(i, j) \in E(G)$ are "generic", that is, there is no nontrivial algebraic relation on all of them. It is easy to see that $G$ has no trivial components.

We proceed by induction on $n$. If $n=2$, then both $a_{12}$ and $a_{21}$ are nonzero, so that $A_{G}$ is invertible, and the assertion of Proposition 4.1 is obvious.

Assume that $n \geq 3$. If $G$ has a vertex of degree one, then, up to relabeling the vertices, we may assume that this vertex is 1 , and that it is incident only with 2 . In this case, if $\mathbf{v}$ is a nullvector of $A_{G}$, then the first row of the matrix equation $A_{G} \cdot \mathbf{v}^{T}=0$ is simply the equation $a_{12} v_{2}=0$, which implies that $v_{2}=0$ because $a_{12} \neq 0$. From now on, we assume that every vertex has degree at least two. 
Assume that $G$ is not connected, and write $G=G_{1} \cup G_{2}$, where $G_{1}$ and $G_{2}$ are two subgraphs of $G$ having $n_{1}$ and $n_{2}$ vertices, respectively, where $n_{1}+n_{2}=n$, and such that there is no edge from a vertex of $G_{1}$ to a vertex of $G_{2}$. Relabeling the vertices, if necessary, we may assume that the first $n_{1}$ vertices are in $G_{1}$ and the remaining $n_{2}$ vertices are in $G_{2}$. Then $A_{G}$ is a block diagonal matrix having $A_{G_{1}}$ and $A_{G_{2}}$ on the main diagonal and zeros in the remaining entries. Write $\mathbf{v} \in V$ as $\mathbf{v}=\left(\mathbf{v}_{1}, \mathbf{v}_{2}\right)$, where $\mathbf{v}_{1}$ stands for the vector constructed with the first $n_{1}$ components of $\mathbf{v}$, and $\mathbf{v}_{2}$ stands for the vector constructed with the last $n_{2}$ components of $\mathbf{v}$. Now whenever $\mathbf{v} \in \operatorname{Ker}(A)$, we get that $\mathbf{v}_{\ell} \in \operatorname{Ker}\left(A_{G_{\ell}}\right)$ for $\ell=1,2$, and, by the induction hypothesis, we get that $\mathbf{v} \in V_{i}$ holds for at least two values of $i$.

We now assume that $G$ is connected, and we let $\Delta_{n}(G)$ be the determinant of $A_{G}$. If $\Delta_{n}(G) \neq 0$, then $A_{G}$ is invertible, and the assertion of Proposition 4.1 is obvious. Assume now that $\Delta_{n}(G)=0$. Before we go on, we make a relevant comment. Given any matrix $A$ with entries in some field, the structure of the nullspace of $A$, and, in particular, whether or not a certain component of an arbitrary vector from the nullspace of $A$ must vanish identically or not, depends on whether or not certain sub-determinants of $A$ vanish. From the way we have defined our matrices $A \in A_{n, \mathcal{N}}$, any given sub-determinant of $A$ that will vanish will do so only because of the location of the zero entries inside this sub-determinant. In particular, the vanishing of such sub-determinants is a property of $G$ itself (or, equivalently, of $\mathcal{N}$ ), and does not depend on any specialization of the entries $a_{i j}$. Thus, whenever we say that a certain sub-determinant vanishes, it simply means that it vanishes identically regarded as a polynomial in its entries not belonging to $\mathcal{N}$.

Let $r$ be the largest positive integer so that $G$ contains a subgraph $G_{r}$ with $\Delta_{r}\left(G_{r}\right) \neq$ 0 . Relabeling the vertices, we may assume that the vertices of $G_{r}$ are $1, \ldots, r$. Write $A_{r}$ for $A_{G_{r}}$, which is the $r \times r$ submatrix of $A$ appearing in the upper left corner. We note that $r \geq 3$. Indeed, since every vertex of $G$ has degree at least two and $G$ is connected, it follows that $G$ has a cycle of length $k \geq 3$. But if $C_{k}$ is the cycle of length $k$, then

$$
A_{C_{k}}=\left(\begin{array}{llllll}
0 & a_{12} & 0 & \ldots & 0 & a_{1 k} \\
a_{21} & 0 & a_{23} & \ldots & 0 & 0 \\
0 & a_{32} & 0 & \ldots & 0 & 0 \\
\ldots & \ldots & \ldots & \ldots & \ldots & \ldots \\
a_{k 1} & 0 & 0 & \ldots & a_{k k-1} & 0
\end{array}\right)
$$

and it is easily seen that $\Delta_{k}\left(A_{C_{k}}\right) \neq 0$. Thus, $r \geq 3$.

Assume first that $r=n-1$. Then the vertex $n$ must be adjacent with some vertex $i \leq r$, and relabeling the first $n-1$ vertices we may assume that $n$ is adjacent to 1 . Let $\Delta_{1 n}$ be the $(n-1) \times(n-1)$ determinant obtained by replacing the first column of $A_{n-1}$ by the column of the first $n-1$ entries (counted from the top towards the bottom) of the $n$th column. We claim that $\Delta_{1 n}=0$. Indeed, to see why this is so, note that if we expand $\Delta_{n}:=\Delta_{n}(G)$ using the last row, we see that $(-1)^{n+1} a_{n 1} \Delta_{1 n}$ is a sub-polynomial of $\Delta_{n}$. Thus, if $\Delta_{1 n} \neq 0$, we get that $\Delta_{n} \neq 0$, which is a contradiction. 
Using this observation, let $\mathbf{v} \in \operatorname{Ker}\left(A_{G}\right)$, and write the system of equations

$$
\sum_{j=1}^{n} a_{i j} v_{j}=0 \quad \text { for } i=1, \ldots, n \text {. }
$$

Disregard the last equation and rewrite the first $n-1$ equations as

$$
\sum_{j=1}^{n-1} a_{i j} v_{i}=-a_{i n} v_{n} \quad \text { for } i=1, \ldots, n-1
$$

The coefficient matrix of the above system is $A_{n-1}$ whose determinant is $\Delta_{n-1}:=$ $\Delta_{n-1}\left(G_{n-1}\right) \neq 0$, and so we may solve this system using Cramer's rule and get

$$
v_{1}=-\frac{\Delta_{1 n}}{\Delta_{n-1}} \cdot v_{n}=0,
$$

which is the assertion of Proposition 4.1.

From now on, we assume that $3 \leq r \leq n-2$. Write $\Delta_{r}:=\Delta_{r}\left(G_{r}\right)$. We first note that if $j>r$, then $j$ is adjacent only with vertices $i \leq r$. Indeed, assume that this is not so. Then up to relabeling the last $n-r$ vertices, we may assume that $r+1$ is adjacent to $r+2$. But then it is easy to see that the nonzero polynomial $a_{r+1, r+2} a_{r+2, r+1} \Delta_{r}$ is a sub-polynomial of the sub-determinant of $A$ formed by the first $r+2$ rows and columns, which is precisely $\Delta_{r+2}\left(G_{r+2}\right)$, where we write $G_{r+2}$ for the subgraph of $G$ spanned by the first $r+2$ vertices and the edges between them. Thus, $\Delta_{r+2}\left(G_{r+2}\right) \neq 0$, which contradicts our definition of $r$. In particular, it follows that $A$ is of the form

$$
A:=\left(\begin{array}{cc}
A_{r} & B \\
C & 0
\end{array}\right)
$$

where $B$ and $C$ are matrices of type $r \times(n-r)$ and $(n-r) \times r$, respectively, whose nonzero entries encode the incidences of the vertices $j>r$ with the vertices of $G_{r}$, and the 0 appearing in the right lower corner in (4.2) above is the $(n-r) \times(n-r)$ zero matrix. Let $\mathbf{v} \in \operatorname{Ker}(A)$, and write $\mathbf{v}=\left(\mathbf{v}_{r}, \mathbf{v}_{n-r}\right)$, where $\mathbf{v}_{r}$ is the vector formed with the first $r$ components of $\mathbf{v}$, and $\mathbf{v}_{n-r}$ is the vector formed with the last $n-r$ components of $\mathbf{v}$. Then, the fact that $\mathbf{v} \in \operatorname{Ker}(A)$ is equivalent to the following system of equations

$$
A_{r} \cdot \mathbf{v}_{r}^{T}=-B \cdot \mathbf{v}_{n-r}^{T} \quad \text { and } \quad C \cdot \mathbf{v}_{r}^{T}=0 .
$$

We take a look at the first equation above. For $i \leq r$ and $j>r$ we write $\Delta_{i j}$ for the determinant of order $r$ of the matrix obtained from $A_{r}$ by replacing the $i$ th column of $A_{r}$ by the $(j-r)$ th column of $B$. Since $A_{r}$ is invertible, we may solve the first Eq. (4.3), either formally to get

$$
\mathbf{v}_{r}^{T}=-\left(A_{r}^{-1} B\right) \cdot \mathbf{v}_{n-r}^{T}
$$


or specifically, using Cramer's rule, to get

$$
v_{i}=-\sum_{j>r} \frac{\Delta_{i j}}{\Delta_{r}} \cdot v_{j} \quad \text { for } i=1, \ldots, r
$$

Fix $i \leq r$. The argument employed in the case $r=n-1$ now shows that if $j>r$ and $\{i, j\} \in E(G)$, then $\Delta_{i j}=0$. Indeed, the argument there was that $a_{j i} \Delta_{i j}$ is a subpolynomial of $\Delta_{r+1}\left(G_{r+1}\right)$, where $G_{r+1}$ is the subgraph of $G$ spanned by $G_{r}$, the vertex $j$, and the edges from $j$ to the vertices of $G_{r}$, and by the definition of $r$ the polynomial $\Delta_{r+1}\left(G_{r+1}\right)$ is identically zero, and therefore $\Delta_{i j}=0$. Thus, if for some $i$ we have that $\Delta_{i j}=0$ for all $j>r$ (and, by what we have just said, this will happen if, say, $i$ is adjacent to all $j>r$ ), we would then get, by (4.5), that $v_{i}=0$, which is the assertion of Proposition 4.1. From now on, we assume that for all $i \leq r$ there exists $j>r$, necessarily not adjacent to $i$, such that $\Delta_{i j} \neq 0$. We now remark that it is also the case that for all $j>r$ there exists $i \leq r$ such that $\Delta_{i j} \neq 0$. Indeed, let $\mathbf{c}_{i}$ be the $i$ th column of $A_{r}$ if $i \leq r$, and the $(i-r)$ th column of $B$ if $i>r$. Since $A_{r}$ is non-degenerate, it follows that for all $j>r$ there exist $\lambda_{i j}$ with $i=1, \ldots, r$ such that

$$
\mathbf{c}_{j}=\sum_{i=1}^{r} \lambda_{i j} \mathbf{c}_{i}
$$

Here, the coefficients $\lambda_{i j}$ are just rational functions in the entries of the $c_{i}$ for $i<r$ and $c_{j}$ not in $\mathcal{N}$ of the $\mathbf{c}_{i}$ for $i \leq r$ and $\mathbf{c}_{j}$. Now the condition $\Delta_{i j}=0$ is equivalent to the fact that $\lambda_{i j}=0$ because the vectors $\mathbf{c}_{i}$ for $i \leq r$ are linearly independent. Thus, should $\Delta_{i j}=0$ held for all $i \leq r$, we would learn that $\mathbf{c}_{j}=0$, which means that $j$ is an isolated vertex, which is a contradiction.

There is a lot more we can learn from relation (4.6). For example, we can learn that the degree of every vertex $j>r$ is $\leq\lfloor r / 2\rfloor$. Indeed, to see why, assume that it is not so, and let $j>r$ be a vertex of degree $t>\lfloor r / 2\rfloor$. By relabeling the vertices of $G_{r}$, we may assume that $j$ is incident to $1,2, \ldots, t$. The previous argument now shows that $\lambda_{i j}=0$ for $i=1, \ldots, t$, therefore relation (4.6) becomes

$$
\mathbf{c}_{j}=\sum_{i>t} \lambda_{i j} \mathbf{c}_{i}
$$

We now see that the right hand side of (4.7) is a linear combination of $r-t<t$ fixed vectors $\mathbf{c}_{i}$. However, the vector $\mathbf{c}_{j}$ contains $t>r-t$ nonzero entries which are algebraically independent over the entries of $A_{r}$, and this is impossible. Thus, $t \leq\lfloor r / 2\rfloor$, and since $t \geq 2$, it follows that in this case we even know that $r \geq 4$.

We now compare (4.4) with (4.5) to infer that $\Delta_{i j} / \Delta_{r}$ is the $i j$ entry of $A_{r}^{-1} B$. Substituting now (4.4) into the second Eq. (4.3), we get

$$
\left(C A_{r}^{-1} B\right) \cdot \mathbf{v}_{n-r}^{T}=0 .
$$

Thus, $\mathbf{v}_{n-r}$ is a null vector of $D:=C A_{r}^{-1} B$ which is a square matrix of order $n-r<n$. To complete the induction, we shall show that $D \in A_{n-r}(\mathbf{K})$.

Springer 
To check conditions 1 and 3 from the definition of $A_{n-r}(\mathbf{K})$, note that if $j>r$, then the $(j-r)$ th row of $D$ is obtained by taking the equation

$$
\sum_{\substack{i \leq r \\\{i, j\} \in E(G)}} a_{j i} v_{i} .
$$

replacing each one of the $v_{i}$ 's by the linear combination shown in (4.5), and then grouping alike terms i.e., grouping all the terms which are multiples of $v_{s}$ for some $s$. Thus, this row is the normal vector to

$$
\sum_{s>r}\left(\sum_{\substack{i \leq r \\\{i, j\} \in E(G)}} \frac{\Delta_{i s}}{\Delta_{r}} a_{j i}\right) v_{s} .
$$

Hence, the nonzero entries of $D$ on row $j-r$ are precisely the entries $d_{j s}$ for which there exists a "mixed" path $(j, i, s)$ of length two with $i \leq r$, such that $j$ is adjacent to $i$ and $\Delta_{i s}$ is nonzero. At least one such entry exists because $j$ is adjacent to some $i \leq r$, and then for this $i$ there exists $s$, necessarily not adjacent to $i$ (and so this $s$ cannot be $j$ ), such that $\Delta_{i s} \neq 0$. This proves that every row of $D$ contains a nonzero entry, and that all the diagonal entries of $D$ are zero.

To prove 2 , note that

$$
D^{T}=\left(C A_{r}^{-1} B\right)^{T}=B^{T}\left(A_{r}^{-1}\right)^{T} C^{T}=B^{T}\left(A_{r}^{T}\right)^{-1} C^{T} .
$$

Let $\mathcal{N}_{1}, \mathcal{N}_{2}$, and $\mathcal{N}_{3}$ be the subsets of $[n-r] \times[r],[r] \times[r]$, and $[r] \times[n-r]$, that are induced by $\mathcal{N}$, and which give the locations of the zero entries in $C, A_{r}$ and $B$, respectively. Note that these subsets are uniquely determined by $\mathcal{N}$, and so they are independent of the matrix $A=A_{G} \in A_{n, \mathcal{N}}$. Let $\mathcal{N}_{4}$ be the subset of $[r] \times[r]$ which gives the locations of the zeros in $A_{r}^{-1}$, whenever $A_{r} \in A_{r, \mathcal{N}_{2}}$. Note that $\mathcal{N}_{4}$ also depends only on $\mathcal{N}$. Finally, write $\mathcal{C}_{\mathcal{N}_{1}}$ (and $\mathcal{B}_{\mathcal{N}_{3}}$, respectively) for the subsets of all matrices in $M_{n-r, r}(\mathbf{K})$ (and $M_{r, n-r}(\mathbf{K})$, respectively) which have the property that their entries in the positions $r s$ for $(r, s) \in \mathcal{N}_{1}$ (and $(r, s) \in \mathcal{N}_{3}$, respectively) are zero, and the remaining entries are algebraically independent over k. Finally, let $\mathcal{N}_{5}$ be the set of zero entries of all the matrices of the type $C^{\prime} A^{\prime} B^{\prime}$ for $C^{\prime} \in \mathcal{C}_{\mathcal{N}_{1}}, A^{\prime} \in A_{r, \mathcal{N}_{4}}$, $B^{\prime} \in \mathcal{B}_{\mathcal{N}_{3}}$ which furthermore have the property that all nonzero entries of $C^{\prime}, A^{\prime}, B^{\prime}$ are algebraically independent over $\mathbf{k}$. It is clear that $\mathcal{N}_{5}$ depends only on $\mathcal{N}$. Now the point is that by (4.9) and the fact that $\mathcal{N}$ is symmetric, it follows that $\left(A_{r}^{T}\right)^{-1}$ is in $A_{r, \mathcal{N}_{4}}$ (like $A_{r}^{-1}$ ), $C^{T} \in \mathcal{B}_{\mathcal{N}_{3}}$ (like $B$ ), and $B^{T} \in \mathcal{C}_{\mathcal{N}_{1}}$ (like $C$ ), and so both $D$ and $D^{T}$ have their zero entries located in precisely the same positions, which proves 2 .

Finally, to prove 4 , write $D:=C\left(A_{r}^{-1} B\right)$. If we write it this way and perform the multiplication, we recognize easily that if $j>r$, then every nonzero entry from $(j-r)$ th row of $D$ is a polynomial which effectively depends on some variable $a_{j i}$ with $i \leq r$, and that the variables of the form $a_{j i}$ with $i \leq r$ did not effectively appear in any of the previous rows of $D$. If we now write $D=\left(C A^{-1}\right) B$, and perform the multiplication, we note that if $j>r$, then every nonzero entry in the column $j-r$ of $D$ involves a variable $a_{i j}$ for $i \leq r$ which did not effectively appear in any of the 
previous columns of $D$. But this is enough to justify that indeed all the nonzero entries of $D$ are algebraically independent over $\mathbf{k}$, which proves 4 . This completes the proof of Proposition 4.1 .

Remark 4.2. From the above proof, we saw that $r \geq 2$ always, and that $r \geq 3$ whenever $G$ is not a union of trees. One can ask if $r$ can be very small. The answer is of course yes, and here is a canonical example. Let $G$ be a bipartite graph. Assume that the partition of $[n]$ is $I \cup J$, where $|I| \leq|J|$, and assume that the first $|I|$ vertices are in $I$ and the remaining ones are in $J$. Then, $A_{G}$ is of the form $\left(\begin{array}{ll}0 & B \\ C & 0\end{array}\right)$ where $B$ and $C$ are matrices of types $|I| \times(n-|I|)$ and $(n-|I|) \times|I|$, respectively, and the upper and lower 0 's stand for the zero square matrices of orders $|I| \times|I|$ and $(n-|I|) \times(n-|I|)$, respectively. It is now very easy to see that the rank of such a matrix cannot exceed $2|I|$, and, in particular, $r \leq 2|I|$. For example, a "star" graph, i.e., the graph such that 1 is adjacent to all other vertices and there are no other edges, has $I=\{1\}$ and $r=2$, while the graph whose set of edges is $\{\{1, j\},\{2, j\} \mid j=3, \ldots, n\}$ has $I=\{1,2\}$ and $r=4$.

The simpler proof of Proposition 4.1 below was communicated to us by Andreas Dress. It is shorter than the first proof above, but we included that proof because of its relationship to the polynomial $P_{G}$ which appears in Section 5 .

Alternative Proof of Proposition 4.1: This proof is a combination of four steps.

1. For the purpose of this proof only, assume that $G^{\prime}$ is an oriented finite and bipartite graph with vertex set $V\left(G^{\prime}\right)=V_{1} \cup V_{2}$ and edge set $E\left(G^{\prime}\right)$ any subset of $V_{1} \times V_{2}$. Then there exists, in view of the celebrated Marriage Theorem (see, for example, [2]), an injective mapping $p: V_{1} \longrightarrow V_{2}$ with $(v, p(v)) \in E\left(G^{\prime}\right)$ for all $v \in V_{1}$, if and only if the set

$$
N_{G^{\prime}}(U):=\left\{v \in V_{2}:(u, v) \in E\left(G^{\prime}\right) \text { for some } u \in U\right\}
$$

has cardinality at least $|U|$ for all finite subsets $U$ of $V_{1}$.

2. Assume now that $A=\left(a_{u, v}: u \in V_{1}, v \in V_{2}\right)$ is a $\left|V_{1}\right| \times\left|V_{2}\right|$ matrix with entries in some field $\mathbf{K}$ such that the following three conditions are satisfied:

(i) $a_{u, v}=0$ if and only if $(u, v) \notin E\left(G^{\prime}\right)$;

(ii) the nonzero entries of $A$ form a family of elements in $\mathbf{K}$ which are algebraically independent over the prime subfield $\mathbf{k} \subset \mathbf{K}$ of $\mathbf{K}$;

(iii) $N(v) \neq \varnothing$ holds for all $v \in V\left(G^{\prime}\right)$.

Assume further that there exists some nonempty finite subset $U$ in $V_{1}$ with $|U|$ at least as large as $|N(U)|$ (for example, we may take $U=V_{1}$ if $\left|V_{1}\right|=\left|V_{2}\right|$ holds), and let $f: V_{2} \longrightarrow \mathbf{K}$ be a map such that the sum

$$
A f(u):=\sum_{v \in V_{2}} a_{u, v} f(v)
$$

vanishes for all $u \in V_{1}$. We then claim that $f(v)=0$ must hold for some $v \in V_{2}$. 
3. Indeed, to prove of the above claim, we choose the smallest such subset $U$ of $V_{1}$, and we then see that the rank of the matrix

$$
A(U, N(U)):=\left(a_{u, v}: u \in U, v \in N(U)\right)
$$

must not only be smaller than or equal to $\min (|N(U)|,|U|)=|N(U)|$, but it also cannot be smaller than $|U|$, because, by 1 above, there must exist, for any subset $W$ of $U$, an injective map $p^{\prime}$ from $W$ into the subset $N(W)$ of $N(U)$ with $\left(w, p^{\prime}(w)\right) \in$ $E\left(G^{\prime}\right)$ for all $w \in W$, which, via condition (ii) from 2 above, implies that the determinant of the sub-matrix $A\left(W, p^{\prime}(W)\right)$ does not vanish. Thus, we get the chain of inequalities

$$
|U| \leq \operatorname{rank}(A(U, N(U))) \leq|N(U)| \leq|U|
$$

and hence, $|U|=\operatorname{rank}(A(U, N(U)))=|N(U)|$. In particular, the determinant of the square matrix $A(U, N(U))$ cannot vanish, implying that $f(u)=0$ must hold for all $u \in U$.

4. One may now start with an arbitrary finite simple graph $G$ with vertex set $V(G)$ and edge set $E(G)$, and with a matrix $A \in A_{n}(\mathbf{K})$, and apply the formalism illustrated in 1-3 above to the bipartite graph $G^{\prime}$ whose vertex set is $V(G) \times\{1,2\}$, edge set is $\{\{\{u, 1\},\{v, 1\}\}:\{u, v\} \in E(G)\}$, matrix $A$, and function $f: V(G) \times\{2\} \longrightarrow \mathbf{K}$ given by $f(\{i, 2\})=v_{i}$, where $\mathbf{v}=\left(v_{1}, \ldots, v_{n}\right)$ is a fixed vector in $\operatorname{Ker}(A)$, to get the conclusion of Proposition 4.1.

\section{A universal polynomial detecting $Z C K$ associated to the matrix of a graph}

The only result of this section is the following:

Proposition 5.1. Let $G$ be a simple graph with $n$ vertices labeled $1,2, \ldots, n$ which has no trivial components. Let $m$ be the number of edges of $G$. Let $A_{G}$ be the matrix whose rows are the normal vectors of the form $h_{i}$ shown in (1.5), where $a_{i j}$ are integers for $\{i, j\} \in E(G)$. Then, there exists a nonzero homogeneous polynomial $P_{G}$ in $2 m$ variables with integer coefficients and degree $<2^{n}$ such that whenever $\mathbf{a}:=$ $\left(a_{i j}\right)_{\{i, j\} \in E(G)} \in \mathbf{Z}^{2 m}$ is not a zero of $P_{G}$, then every nullvector $\mathbf{v}:=\left(v_{1}, \ldots, v_{n}\right) \in \mathbf{Q}^{n}$ of $A_{G}$ has the property that $v_{i}=0$ holds for some $i \leq n$.

Proof of Proposition 5.1: Except for the statement about the degree of $P_{G}$, the assertion of Proposition 5.1 can be read off from the proof of Proposition 4.1.

We proceed to construct $P_{G}$ by induction on $n$. If $n=2$, we may simply take $P_{G}:=\Delta_{2}(G)=-a_{12} a_{21}$, and now if $\mathbf{v} \in \mathbf{Q}^{2}$ is a nullvector of $A_{G}$, then $v_{1}=v_{2}=0$ whenever $P_{G}(\mathbf{a}) \neq 0$. The degree of $P_{G}$ is $2<2^{2}$.

Assume that $n \geq 3$. If there exists a vertex $i$ of $G$ of degree 1 , we may relabel the vertices of $G$, and assume that this vertex is 1 , and that it is adjacent only to 2 . We may then take again $P_{G}:=-a_{12} a_{21}$, which is of degree $2<2^{n}$, and then every nullvector $\mathbf{v} \in \mathbf{Q}^{n}$ of $A_{G}$ has $v_{2}=0$ provided that $P_{G}(\mathbf{a}) \neq 0$. 
Assume now that every vertex of $G$ has degree at least two. If $G$ is not connected, let $G_{1}$ be a connected component of $G$ with $n_{1}<n$ vertices. We may assume that the vertices of $G_{1}$ are $1,2, \ldots, n_{1}$. It is then clear that we can choose $P_{G}:=P_{G_{1}}$, which, by induction, has degree $<2^{n_{1}}<2^{n}$, and then every nullvector $\mathbf{v} \in \mathbf{Q}^{n}$ of $A_{G}$ will have the property that $v_{i}=0$ for some $i \leq n_{1}$.

Assume now that $G$ is connected. If either $\Delta_{n}$ is not zero, or $\Delta_{n}$ is zero but $\Delta_{n-1}$ is nonzero, where these polynomials were defined in the proof of Proposition 4.1, then we can take $P_{G}:=\Delta_{n}$, or $P_{G}:=\Delta_{n-1}$, respectively, which are polynomials of degree $\leq n<2^{n}$, and the argument from the proof of Proposition 4.1 shows that every nullvector $\mathbf{v} \in \mathbf{Q}^{n}$ of $A_{G}$ has $v_{i}=0$ for some $i$.

Assume now that $\Delta_{n}=\Delta_{n-1}=0$, and let $r \leq n-2$ be the number defined in the proof of Proposition 4.1. Recall that $r \geq 3$. The argument from the proof of Proposition 4.1 now shows that we may either take $P_{G}:=\Delta_{r}$, or we may take $P_{G}$ to be the numerator of a rational function of the form

$$
\Delta_{r} \cdot P_{G^{\prime}}\left(f_{1}, \ldots, f_{2 m^{\prime}}\right)
$$

where $G^{\prime}$ is the graph on a set of $n-r$ vertices and $m^{\prime}<m$ edges whose adjacencies are encoded in the nonzero "generic" entries of the matrix $D$ from the proof of Proposition 4.1 , and where $f_{1}, f_{2}, \ldots, f_{2 m^{\prime}}$ are just the rational functions (versus $a_{i j}$ for $\{i, j\} \in$ $E(G)$ ) which appear in the nonzero entries of $D$. All such rational functions $f_{\ell}$ for $\ell=1,2, \ldots, 2 m^{\prime}$ are of the type $P_{\ell} / \Delta_{r}$, where $P_{\ell}$ is a homogeneous polynomial of degree exactly $r+1$ and, in fact, every such $f_{\ell}$ is a sum of rational functions of the form $a_{j i} \cdot \frac{\Delta_{i s}}{\Delta_{r}}$ for $j>r, i \leq r$, and $s>r$. Since $P_{G^{\prime}}$ is homogeneous, we may discard the common denominator $\Delta_{r}$ of all the $f_{\ell}$ 's, and infer that

$$
\operatorname{deg}\left(P_{G}\right) \leq \operatorname{deg}\left(\Delta_{r}\right)+(r+1) \operatorname{deg}\left(P_{G^{\prime}}\right)<r+(r+1) 2^{n-r}<(2 r+1) 2^{n-r}<2^{n},
$$

where the last inequality holds because it is equivalent to $2 r+1<2^{r}$, which is indeed fulfilled whenever $r \geq 3$. Proposition 5.1 is therefore proved.

\section{Proof of Theorem 1.2}

This is almost immediate. Let $G$ be a graph with $n$ vertices and $m$ edges. Let $A:=A_{G}$ be a matrix associated to $G$ in such a way that the $i$ th row of $A$ is the normal vector to $h_{i}$ for $i=1, \ldots, n$, where the forms $h_{1}, \ldots, h_{n}$ are the ones appearing in (1.5). Assume that $a_{i j} \in \mathbf{Z}^{*}$ and write $\mathbf{a}:=\left(a_{i j} \mid\{i, j\} \in E(G)\right) \in \mathbf{Z}^{2 m}$.

Let $H$ be any nonempty subgraph of $G$ without isolated vertices. Write $n_{H}:=$ $|V(H)|$ and $m_{H}:=|E(H)|$. Proposition 5.1 shows that there exists a nonzero polynomial $P_{H} \in \mathbf{Z}^{2 m_{H}}$ of degree $<2^{n_{H}} \leq 2^{n}$ such that if $\mathbf{a} \downarrow_{H}^{G}:=\left(a_{i j} \mid(i, j) \in\right.$ $E(H)) \in \mathbf{Z}^{2 m_{H}}$ is such that $P_{H}\left(\mathbf{a} \downarrow_{H}^{G}\right) \neq 0$, then $A \downarrow_{H}^{G}$ is $Z C K$. Let

$$
R_{G}:=\prod_{H \subseteq G} P_{H} \in \mathbf{Z}\left[X_{i j} \mid\{i, j\} \in E(G)\right],
$$


where in the above product $H$ runs over all the nonempty subgraphs of $G$ without isolated vertices. We conclude that if $\mathbf{a} \in \mathbf{Z}^{2 m}$ is such that $R_{G}(\mathbf{a}) \neq 0$, then $A$ is $H Z C K$. Note that at this point we no longer need to impose the restriction that $a_{i j} \in \mathbf{Z}^{*}$, because for any $e:=\{i, j\} \in E(G)$ both indeterminates $X_{i j}$ and $X_{j i}$ are factors of $R_{G}$. Indeed, this follows by noting that when $H$ is the subgraph with $V(H):=\{i, j\}$ and $E(H):=\{e\}$, then $P_{H} \in \mathbf{Z}\left[X_{i j}, X_{j i}\right]$ is precisely $\Delta_{2}(H)=-X_{i j}$. $X_{j i}$.

Since $G$ cannot possibly contain more than $2^{m}$ subgraphs $H$ (note that $2^{m}$ is the cardinality of the power set of $E(G)$ ), it follows that the degree of $R_{G}$ is smaller than $2^{n+m}$. We now recall without proof the following elementary fact.

Lemma 6.1. Let $M$ be a positive integer, and let $R$ be a nonzero polynomial with integer coefficients in $t \geq 1$ variables $X_{1}, \ldots, X_{t}$ of total degree $<M$. Then, there exists $\mathbf{x}:=\left(x_{1}, \ldots, x_{t}\right) \in \mathbf{Z}^{t}$ with $1 \leq x_{i} \leq M$ for all $i=1, \ldots, t$ such that $P(\mathbf{x}) \neq 0$.

Returning to our proof of Theorem 1.2, we conclude that there exists a vector $\mathbf{a} \in \mathbf{Z}^{2 m}$, such that $1 \leq a_{i j} \leq 2^{n+m}$ for all $\{i, j\} \in E(G)$, and such that $R_{G}(\mathbf{a}) \neq 0$. For such a value of a, the matrix $A$ is $H Z C K$, and by Proposition 3.3 we conclude that $h_{1}, \ldots, h_{n}$ fulfill the conditions of Question 1.1. This completes the proof of Theorem 1.2.

\section{Comments, remarks and the case of finite characteristic}

1. In [1], Alcántar lets $G:=\mathcal{K}_{n}$ be the complete graph on $n$-vertices, and shows that if one takes $a_{i j}:=1$ for all $i \neq j$, then the forms $h_{i}$ given by (1.5) do provide a standard Noether normalization of $\mathbf{k}[G]$, provided that $\mathbf{k}$ is either of characteristic zero, or of positive characteristic $>n-1$. His proof of this result uses the precise description of $P(G)$ for such a graph $G$ which he inferred from [3] and [6]. Here, we give an elementary proof of Alcántar's result, assuming, of course, that $\mathbf{k}[G]$ has dimension $n$. With our formalism and Alcántar's choice of coefficients, we have

$$
A_{G}:=A_{n}:=\left(\begin{array}{ccccc}
0 & 1 & 1 & \ldots & 1 \\
1 & 0 & 1 & \ldots & 1 \\
1 & 1 & 0 & \ldots & 1 \\
& \ldots & \ldots & \ldots & \\
1 & 1 & 1 & \ldots & 0
\end{array}\right)
$$

and the determinant of $A_{n}$ is $n-1$, a fact which can be easily shown either by induction, or simply by using a classical formula for the determinant of a circulant matrix. Thus, if $\mathbf{k}$ is any field of characteristic zero, or of positive characteristic $>n-1$, then $A_{n}$ is non-degenerate, and therefore any solution $\mathbf{v} \in \mathbf{k}^{n}$ of the system of Eq. (3.3) (with $\overline{x_{i}}:=v_{i}$ for $i=1, \ldots, n$ ) must have $v_{i}=0$ for some $i$. Eliminating the vertex $i$ and the edges adjacent to it, we get $G \backslash\{i\}=\mathcal{K}_{n-1}$, and $A_{G \backslash\{i\}}=A_{n-1}$. Thus, we may continue by successively eliminating vertices to obtain smaller complete graphs, and conclude that if $\mathbf{k}$ is of characteristic zero, or of positive characteristic $>n-1$, then any solution $\mathbf{v} \in \mathbf{k}^{n}$ of the system of 
Eq. (3.3) has $n-1$ of its components equal to zero. The argument from the proof of Proposition 3.3 now shows that if $P$ is a prime ideal in $S$ containing $P(G)$ and $h_{i}$ for all $i=1, \ldots, n$, then there must exist an edge $e \in E(G)$ such that $t_{e} \in P$. Assume that $e:=\{1,2\}$. If all the edge indeterminates adjacent to 1 belong to $P$, then we may eliminate vertex 1 and all the edges adjacent to it and reduce the problem to the complete graph $\mathcal{K}_{n-1}$ with $n-1$ vertices. Let us assume now that there exists an edge adjacent to 1 , say $e^{\prime}:=\{1,3\}$, such that $t_{e^{\prime}} \notin P$. It follows that $n \geq 4$ because if not then $h_{1}=t_{e}+t_{e^{\prime}} \in P$, and $t_{e} \in P$, and therefore $t_{e^{\prime}} \in P$ as well. The element $t_{e} t_{\{3, i\}}-t_{e^{\prime}} t_{\{2, i\}}$ obviously belongs to $P(G)$ for all $i \geq 4$, and since $t_{e} \in P$ and $t_{e^{\prime}} \notin P$, it follows that $t_{\{2, i\}} \in P$ for all $i \geq 4$. Since $t_{e}=t_{\{2, i\}} \in P$ and $h_{2}=\sum_{i \neq 2} t_{\{2, i\}} \in P$, we conclude that all the edge indeterminates of edges adjacent to 2 belong to $P$. Thus, we may eliminate vertex 2 and all the edges adjacent to it and reduce the problem to a smaller complete graph $\mathcal{K}_{n-1}$ with $n-1$ vertices. The result follows now by induction on $n$.

2. One can ask whether or not the matrix shown in (7.1) is $H Z C K$. The answer is no. Indeed, if the matrix shown in (7.1) were $H Z C K$ for all $n \geq 2$, it would then follow that if $G$ is any finite graph without isolated vertices, then the matrix $A_{G}$ associated to the forms $h_{i}$ for $i=1, \ldots, n$, appearing in (1.5) with the choice of coefficients $a_{i j}=1$ for all $\{i, j\} \in E(G)$ would also be $H C Z K$ (because any graph $G$ is a subgraph of $\mathcal{K}_{n}$ with $n=V(G)$ ), and, in particular, by Proposition 3.3, the forms $h_{1}, \ldots, h_{n}$ will fulfill the conditions from Question 1.1. However, Alcántar's Example 4.3 from [1] shows that this is not the case for a certain graph $G$ with $n:=|V(G)|=5$ and $m:=|E(G)|$ $=8$.

3. While the matrix shown in (7.1) is not $H Z C K$, Theorem 1.2 proves that there exists an $H C Z K$ matrix associated to the complete graph $\mathcal{K}_{n}$ whose entries are positive integers $\leq 2^{n(n+1) / 2}$. Detecting such a matrix for a given $n$, or a parametric family of such matrices depending on $n$, could be of interest since such a matrix will provide universal standard Noether normalizations for all graphs with at most $n$ vertices, simply by considering the matrices the $A_{\mathcal{K}_{n}} \downarrow_{G}^{\mathcal{K}_{n}}$ for all such graphs $G$.

4. We also note that since our arguments show that $\mathbf{a}:=\left(a_{i j} \mid\{i, j\} \in E(G)\right) \in \mathbf{Z}^{2 m}$ has the property that the forms shown in (1.5) fulfill the condition from Question 1.1 whenever $R_{G}(\mathbf{a}) \neq 0$, and since algebraic sets are "thin", we conclude that, in practice, when searching for such a vector a, one may simply pick vector a at random, and the chances of this random vector to produce a standard Noether normalization for $G$ are as good as any.

5. One might also ask whether our theorem is optimal with respect to sparseness. That is, does there exist a graph subring $S$ such that the Noether normalization constructed in Theorem 1.2 is the one with the least number of nonzero coefficients? We did not try to answer this question.

Using the observation from 2 above regarding the existence of a standard Noether normalization of the complete graph over a field of positive characteristic which is sufficiently large, we finally provide the proof of Theorem 1.2 in the case when $\mathbf{k}$ is of finite characteristic. The precise result is the following. 
Proposition 7.1. The conclusion of Theorem 1.2 holds over any field $\mathbf{k}$ of positive characteristic $p>2^{2^{n} n^{3}}$.

Proof of Proposition 7.1. It suffices to assume that $n>2$, otherwise Proposition 7.1 holds over any field anyway (for $n=2$ simply take $a_{12}=a_{21}=1$ in (1.5)).

A careful analysis of the arguments used in the proofs of Propositions 4.1 and 5.1 shows that $P_{G}$ is a homogeneous polynomial of degree $d_{G}=\operatorname{deg}\left(P_{G}\right)<2^{n}$ in $2 m$ variables, and that moreover every monomial of total degree $d_{G}$ in the $2 m$ variables appears in the representation of $P_{G}$ with coefficient 0 or \pm 1 , and, of course, at least one of them appears with a nonzero coefficient because $P_{G}$ is not constant zero. This shows that $P_{G}$ is not constant zero over any field. It also shows that the inequality $\left|P_{G}(\mathbf{a})\right| \leq h\left(d_{G}, 2 m\right)|| \mathbf{a}||^{d_{G}}$ holds for all $\mathbf{a} \in \mathbf{Z}^{2 m}$, where we use $\|a\|:=\max \left\{\left|a_{i j}\right|:\right.$ $\mid\{i, j\} \in E(G)\}$, and $h\left(d_{G}, 2 m\right)$ stands for the number of all the monomials of total degree $d_{G}$ in $2 m$ variables. Obviously,

$$
h\left(d_{G}, 2 m\right)<\left(\begin{array}{c}
d_{G}+2 m \\
2 m
\end{array}\right)<\left(d_{G}+2 m\right)^{2 m}<\left(2^{n}+n(n-1)\right)^{n(n-1)} \leq\left(2^{n+1}\right)^{n(n-1)}<2^{n^{3}},
$$

where in the above inequality we used the fact that the inequality $2^{n} \geq n(n-1)$ holds for all $n \geq 2$. Thus, $\left|P_{G}(\mathbf{a})\right| \leq 2^{n^{3}} \| \mathbf{a}||^{2^{n}}$ holds for all $\mathbf{a} \in \mathbf{Z}^{2 m}$. In particular, the polynomial $R_{G}$ given by (6.1) is not the zero polynomial over any field, and whenever $\mathbf{a} \in \mathbf{Z}^{2 m}$ is such that $R(\mathbf{a}) \neq 0$, then $|R(\mathbf{a})|$ is a product of positive integers none of which exceeds $2^{n^{3}}\|a\|^{2^{n}}$. Since by Theorem 1.2, a choice of $\mathbf{a} \in \mathbf{Z}^{2 m}$ with $\|a\| \leq 2^{n+m}$ exists such that $R_{G}(\mathbf{a}) \neq 0$, we conclude that for such an integer vector $\mathbf{a}$, the integer $\left|R_{G}(\mathbf{a})\right|$ is a product of positive integers none of which exceeds

$$
2^{n^{3}}\left(2^{n+m}\right)^{2^{n}} \leq 2^{n^{3}+(n+n(n-1) / 2) \cdot 2^{n}} \leq 2^{n^{2} \cdot 2^{n}}
$$

where in the above inequality (7.2) we used the fact that the inequality

$$
2^{n} \cdot n^{2}>n^{3}+2^{n} \cdot\left(n+\frac{n(n-1)}{2}\right) \geq n^{3}+2^{n}(n+m)
$$

holds for all $n \geq 3$. Thus, if $\mathbf{k}$ is any field of positive characteristic larger than $2^{2^{n} n^{3}}$, then $R_{G}(\mathbf{a})$ is nonzero in $\mathbf{k}$, which completes the proof of Proposition 7.1.

Acknowledgements We thank the anonymous referees, and Adrian Alcántar and Rafael Villarreal for useful comments. Our special thanks go to Andreas Dress for a careful proofreading of a preliminary version of this paper, and for providing a shorter proof of Proposition 4.1. Both authors were partly supported by Grant PAPIIT IN-104602 from the UNAM. The first author was also partly supported by Grant SEP-CONACyT 37260-E, and the second author was also partly supported by Grant SEP-CONACyT J38611-E.

\section{References}

1. A. Alcántar, "Noether normalizations of some subrings of graphs," Comm. Alg. 29 (2001), 5525-5534.

2. A. Dress, "The theorem of the $k-1$ happy divorces," Ann. Comb. 4 (2000), 183-194. 
3. M. Hochster, "Rings of invariants of tori, Cohen-Macaulay rings generated by monomials, and polytopes," Ann. Math. 96 (1972), 318-337.

4. A. Simis, W.V. Vasconcelos, and R. Villarreal, "On the ideal theory of graphs," J. Algebra 167 (1994), $389-416$.

5. A. Simis, W.V. Vasconcelos, and R. Villarreal, "The integral closure of subrings associated to graphs," J. Algebra 199 (1998), 281-289.

6. R. Villarreal, "Normality of subrings generated by squarefree monomials," J. Pure App. Alg. 113 (1996), 91-106.

7. R.H. Villarreal, Monomial Algebras, Monographs and Textbooks in Pure and Applied Mathematics, 238 Marcel Dekker, Inc., New York, 2001. 